\title{
Quasicrystals - A Paradigm Shift in Crystallography?
}

\author{
Walter Steurer*
}

\begin{abstract}
The discovery of quasicrystals had important consequences for our understanding of long-range order in thermodynamic equilibrium, the definition of the term 'crystal' as well as diffraction theory. Quasicrystals have been observed not only at the atomic scale in binary and ternary intermetallic systems, but also at the mesoscale in self-assembled colloids and block-copolymers, and even at the macroscale in packings of hard polyhedra. How important was the discovery of quasicrystals for crystallography? Did it usher in a scientific revolution and a paradigm shift? These questions are discussed following a short overview of the status of quasicrystal research.
\end{abstract}

Keywords: Intermetallics · Paradigm shift · Quasicrystals · Self-assembly

\section{Quasicrystals - A Paradigm Shift in Crystallography?}

"Though the world does not change with a change of paradigm, the scientist afterward works in a different world." [1, p. 121]

They neither changed the world nor did they excite crystallographers all over the globe. It was simply unbelievable, not only for double Nobel laureate Linus Pauling, but also for quite a few educated crystallographers. No wonder that it took a couple of years until it was commonly accepted that the equilibrium state of solid matter does not necessarily have to be periodic, not even on average. As a matter of fact, under specific conditions crystals can be quasiperiodic (Figs. 1, 2).

Shechtman, a mid-career materials scientist at that time, tried for more than two years to get his discovery, made in April 1982, published in a paper entitled 'Metallic Phase with Long-Range Orientational Order and No Translational Symmetry'.[2] Shortly after, this phase, exhibiting icosahedral diffraction symmetry, was named 'quasicrystal' (QC). ${ }^{[3]}$ Shechtman was struggling hard with the disbelief of eminent scientists, who 'knew' that QCs could not exist because this would infringe on fundamental laws of crystallography. Fortunately, Shechtman was hard-nosed enough not to give up firmly believing in his own experimental work.

"Almost always the men who achieve these fundamental inventions of a new paradigm have been either very young or

\footnotetext{
${ }^{*}$ Correspondence: Prof. Dr. W. Steurer

Laboratory of Crystallography

ETH Zurich

Vladimir-Prelog-Weg 10

$\mathrm{CH}-8093$ Zurich

Tel.: + 41446326650

E-mail: steurer@mat.ethz.ch
}

very new to the field whose paradigm they change.”[1, p. 90]

Perhaps, the disbelievers would have been convinced more rapidly had they known of a visionary paper ${ }^{[4]}$ by the famous British crystallographer Alan L. Mackay, who was particularly interested in 'generalized crystallography'. This article was published in August 1982, showing an optical diffraction pattern of a Penrose tiling (Fig. 3, outlined in green). The similarity with Shechtman's tenfold symmetric diffraction pattern ${ }^{[2]}$ is obvious. Mackay introduced the term 'quasi-lattice' and suggested two-dimensional (2D) and three-dimensional (3D) structure models.

Why have crystallographers more or less ignored this challenging field of QCs? Even the Nobel Prize for Chemistry 2011, awarded to Dan Shechtman for his discovery, brought QCs to the attention of the crystallographic community only for a short time. Why have hard-core crystallographers never taken up the challenge to develop a new toolbox for higher-dimensional crystallography and to solve the strange structures of QCs? Why was this opportunity to extend the crystallographic approach to quasicrystals mostly left to a comparably small community of interested physicists, mathematicians and materials scientists?

It seems that most crystallographers have never recognized QCs as 'true crystals' that are worthwhile of being studied, perhaps, because in the first years, QCs could only be prepared by rapid solidification. An analogous observation holds for amorphous metals, for instance. They too were and are studied by a community essentially outside of crystallography. In contrast, $\mathrm{C}_{60}$ with its icosahedral symmetry and fullerenes in general were adopted by crystallographers immediately after their

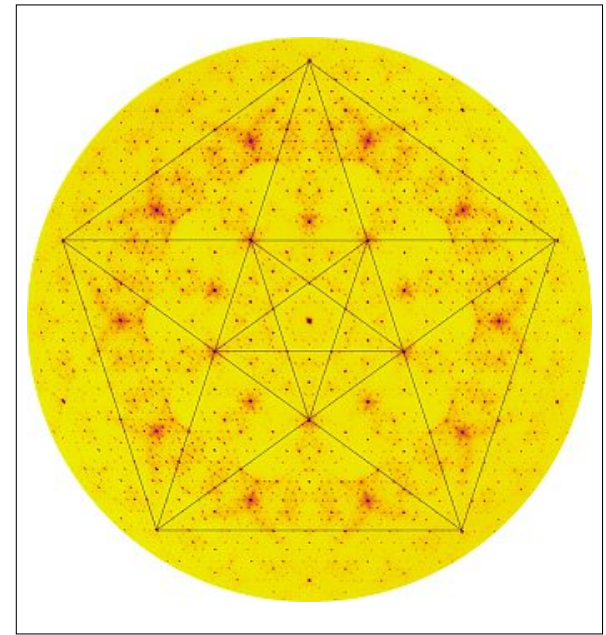

Fig. 1. X-ray diffraction pattern with pentagrammal (and decagrammal) symmetry.

discovery, possibly because their periodic crystal structures, which could be analyzed by well-established methods, made them 'trustworthy crystals'. The crystallographic community has also been less reluctant to deal with the other classes of aperiodic crystals, namely the incommensurately modulated structures and the composite (host/guest) structures. Perhaps, because in contrast to QCs, these aperiodic crystals can still be described based on periodic basic structures and still fit in the framework of classical crystallography.

Before discussing the question whether or not the discovery of QCs ushered in a paradigm shift in crystallography, whether it was a revolutionary rather than an evolutionary discovery, we will give a short review of the actual status of QC research.

"The transition from a paradigm in crisis to a new one from which a new tradition of normal science can emerge is far from a cumulative process, one achieved by an articulation or extension of the old 


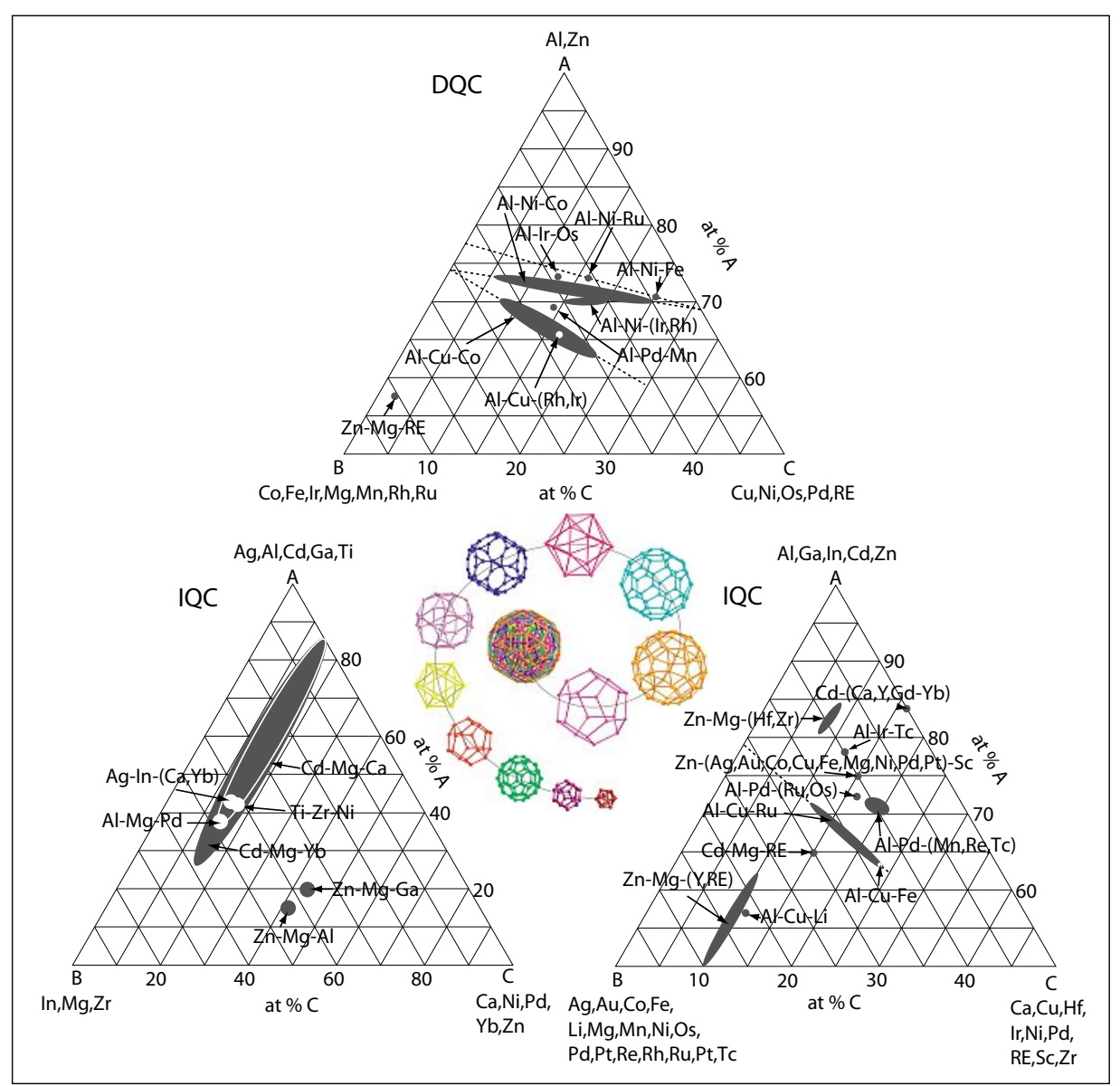

Fig. 2. Concentration diagrams of decagonal and icosahedral QCs. The stability fields are shaded grey. In the center figure, the onion-like shell structure of a typical endohedral QC cluster is illustrated.

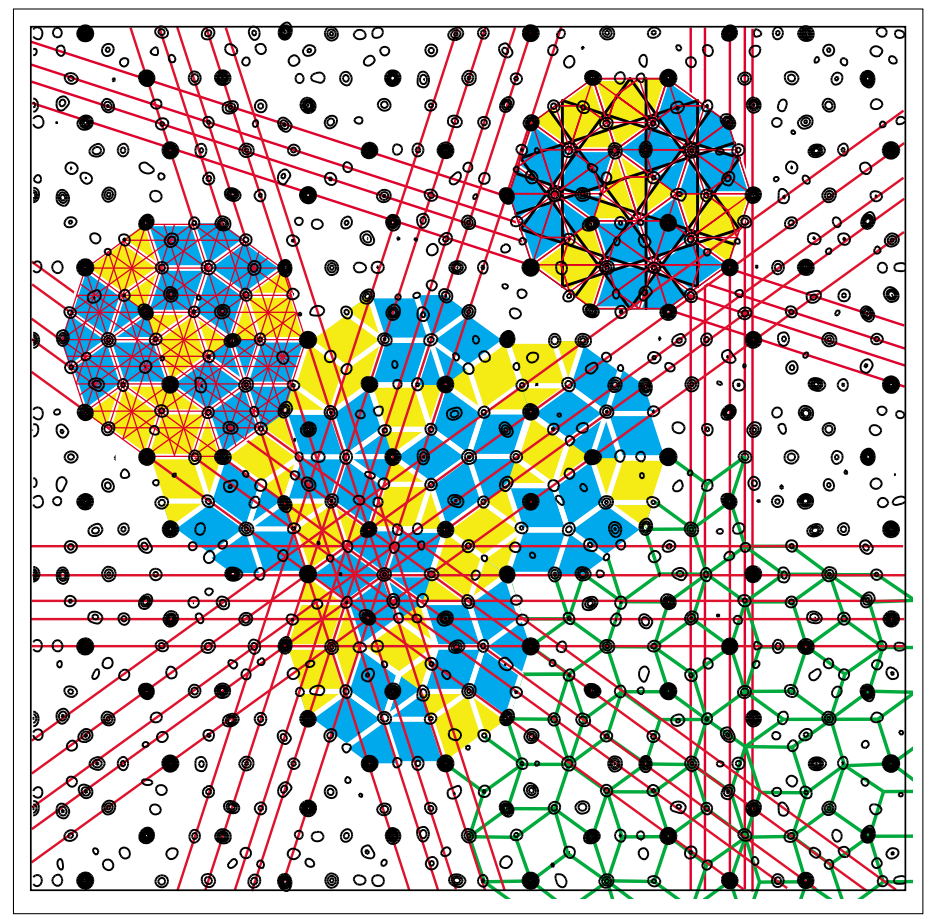

Fig. 3. Electron density map projected along the tenfold axis of decagonal Al-Co-Ni. Gummelt decagons are shaded yellow with the blue parts marking potentially overlapping areas (overlapping rules). If the overlapping rules are obeyed everywhere in the structure then the underlying tiling is strictly quasiperiodic. A patch of a classical Penrose tiling built from two rhombs of different shapes is outlined in green, quasilattice planes in red.

paradigm. Rather it is a reconstruction of the field from new fundamentals, $a$ reconstruction that changes some of the field's most elementary theoretical generalizations as well as many of its paradigm methods and applications. During the transition period there will be a large but never complete overlap between the problems that can be solved by the old and by the new paradigm. But there will also be a decisive difference in the modes of solution. When the transition is complete, the profession will have changed its view of the field, its methods, and its goals."[1, p. 85]
Quasiperiodic structures are nowadays known at different length scales: at the atomic scale in intermetallic phases, at the mesoscale in self-assembled colloids and block-copolymers, and at the macroscale for packings of particular classes of polyhedra (for overviews see, for instance, refs [5-7]). While in the first case decagonal or icosahedral symmetry is predominant, in the other two cases it is the dodecagonal symmetry. It has been shown by model calculations that all these different kinds of quasiperiodic long-range order are stabilized by particular interaction potentials favoring two different length scales. ${ }^{[8]}$ The ratio of the two basic lengths ('atomic' distances) essentially determines the resulting symmetry. Recently, dodecagonal quasiperiodic order has also been found in a thin $\mathrm{BaTiO}_{3}$ film grown on a $\mathrm{Pt}(111)$ surface. ${ }^{\text {[9] }}$ This observation may open up the way for growing heteroepitaxially quasiperiodic thin films of a large class of ionically bonded materials such as multiferroics, for instance.

In the case of intermetallic QCs, electronic stabilization according to the HumeRothery mechanism (Fermi surface/ Brillouin zone nesting) plays an important role, perhaps even a decisive one. Indeed, many QCs have been discovered based on particular values for the valence electron concentration. ${ }^{[10]}$ It is not fully understood yet, whether or not an entropic contribution to their stability is necessary, e.g. a minimum amount of phasonic or other kind of disorder. The assumption of an entropic contribution is favored, however, by indications that QCs are high-temperature phases and do not represent groundstates at zero Kelvin. An important geometric factor favoring the formation of quasiperiodic order is the optimization of the packing of clusters, whose structures can be described as projection of higher-dimensional polytopes. ${ }^{[11]}$ The term 'cluster' should be understood here as synonymous for 'recurrent structural building unit', the chemical bonding between atoms within such a cluster and between atoms belonging to different clusters is of the same kind. ${ }^{[12,13]}$

Thermodynamically stable intermetallic QCs seem to be quite abundant (Fig. 2). They can be grown like 'normal' crystals and can have a high degree of perfection comparable to that of the very best intermetallic compounds. QCs of a size needed for $\mathrm{X}$-ray structure analysis can be easily obtained, and in some cases even centimetersize QCs have been grown. Consequently, much has been learned about the structures of QCs from both single crystal X-ray diffraction as well as from electron microscopy, although the information is always averaged in one way or another. ${ }^{[14]}$ The main problem with QC structure analysis 
is that both the structure of the clusters themselves and the long-range order of the clusters have to be determined. In contrast, for periodic crystals, only the content of one unit cell has to be determined, the identification of the lattice is straightforward. In the case of QCs both the atomic arrangement inside the clusters as well as the arrangement of clusters can be disordered, while we do not need to consider the latter in the case of periodic crystals.

Mesoscopic quasiperiodic structures are simpler to study experimentally and their formation and growth are easier to understand and model. There are no electronic contributions to consider, and only rather simple 'atomic' interactions have to be taken into account. In the case of the self-assembly of micelles in a liquid, for instance, the two necessary length scales are provided by a soft repulsive interaction between the coronas of the micelles and a hard repulsion between their cores. ${ }^{[15-17]}$ Mesoscopic quasiperiodic structures can be modeled successfully even with a simple step-function potential.

A similar mechanism governs the packing of hard tetrahedra, for instance. They order in an arrangement with overall dodecagonal (diffraction) symmetry. Here, the two length scales are provided by the geometry of the polyhedra and the ways they can be arranged (face to face or edge on edge etc.); the resulting quasiperiodic arrangement is solely of entropic origin. [18] This implies that the structure (underlying tiling) is not strictly quasiperiodic, but just on average (random tiling).

Mesoscopic QCs, be they self-assembled or top down manufactured, are of particular interest for applications such as photonic or phononic crystals. ${ }^{[19]}$ Their high symmetry allows for highly isotropic band gaps as well as very versatile defect generation and band-gap engineering.

Quantitative QC structure analysis has been very successful thanks to the power of higher-dimensional ( $n \mathrm{D})$ crystallography. Carl Hermann[20] paved the way for $n \mathrm{D}$ crystallography already in 1949 . He was interested in the question which rotational symmetries were compatible with lattices in $n$ dimensions. For 5-, 8-, 10- and 12-fold axial QCs 4D lattices are needed, and for icosahedral QCs (IQC) 6D lattices, for instance. Powerful computer programs are now available for $n \mathrm{D}$ structure solution based on the charge-flipping and/or low-density-elimination approach (SUPERFLIP[21]) and for $n \mathrm{D}$ structure refinement (QUASI07_08[22]).

Aspects of QC that are less well understood include their formation and growth and the factors favoring quasiperiodic long-range order (for an introduction into this problem, see, for instance, ref. [23] and references therein). A valuable help for the analysis of the latter problem is the existence of approximants to QCs. Approximants are periodic structures built from the same clusters as QCs; their stability regions in phase diagrams are close to those of the related QCs. They are called rational if their structures can be generated using the $n \mathrm{D}$ approach (for an introduction see, for instance, ref. [6]). There is only a small difference between the stoichiometries of QCs and their approximants, if both exist in the same binary or ternary intermetallic system. Consequently, the chemical potential is the most important factor for stabilizing one or the other of the two structures. In some cases, the slightly different electron concentrations resulting from the different chemical compositions may also be important.

One way to describe the structure of QCs is based on the packing of partially overlapping endohedral clusters, which are decaprismatic columnar in the case of decagonal QCs (DQC, see Fig. 3) and of triacontahedral shape in the case of IQCs. The cluster centers decorate the vertices and other special sites of tilings, which show close relationships to $2 \mathrm{D}$ and $3 \mathrm{D}$ Penrose tilings, respectively.

Discussing quasiperiodicity also means discussing the difference to periodicity and asking why periodicity is such a strong ordering principle that it applies to almost all materials which are crystallizable, from rock salt to viruses. Periodicity is the result of maximizing the packingdensity of structural subunits (atomic environment types, AETs) under the constraint of minimizing the Gibbs free energy. This leads to a narrow distribution of atomic (molecular) distances and a minimum of different AETs. Furthermore, structures based on periodic lattices allow a rich phonon spectrum, Bloch waves and a discrete autocorrelation function, because of the finite number of different atomic distances in a given range everywhere in an infinite structure. Even QCs, at least those which have been experimentally observed so far, exhibit a kind of discrete periodic average structures (PAS) ${ }^{[24,25]}$ on sublattices related to strong Fourier coefficients. Consequently, Bloch waves and phonons in QCs may be supported by the different PAS present in each QC. Of course, real crystals are never strictly periodic, because of their finite size, thermal vibrations (phonons), thermal vacancies (equilibrium defects), impurities (point defects), etc. Nevertheless, periodicity is a fruitful concept to understand many properties of crystals and QCs.

Periodicity reduces the complexity in the description of an infinite crystal structure drastically, at least if compared to the complexity of amorphous structures. It is sufficient to know the atoms in the asymmetric unit, the space group symmetry and the Bravais lattice. How does this compare with QCs? Are periodic crystals less complex than quasiperiodic ones, which do not even have a unit cell?

An answer to these questions requires a measure of structural complexity. I will illustrate this with the simple example of the 1D quasiperiodic Fibonacci sequence (FS) and some of its approximants. Each of the three structures in Fig. 4 is fully defined by giving a 2D lattice, a strip with a particular slope and a width defined by one $2 \mathrm{D}$ unit cell that just fits into the strip. $1 \mathrm{D}$ 'crystals' are generated by projecting all lattice points within the strips onto the red lines ('physical space'). Whether the

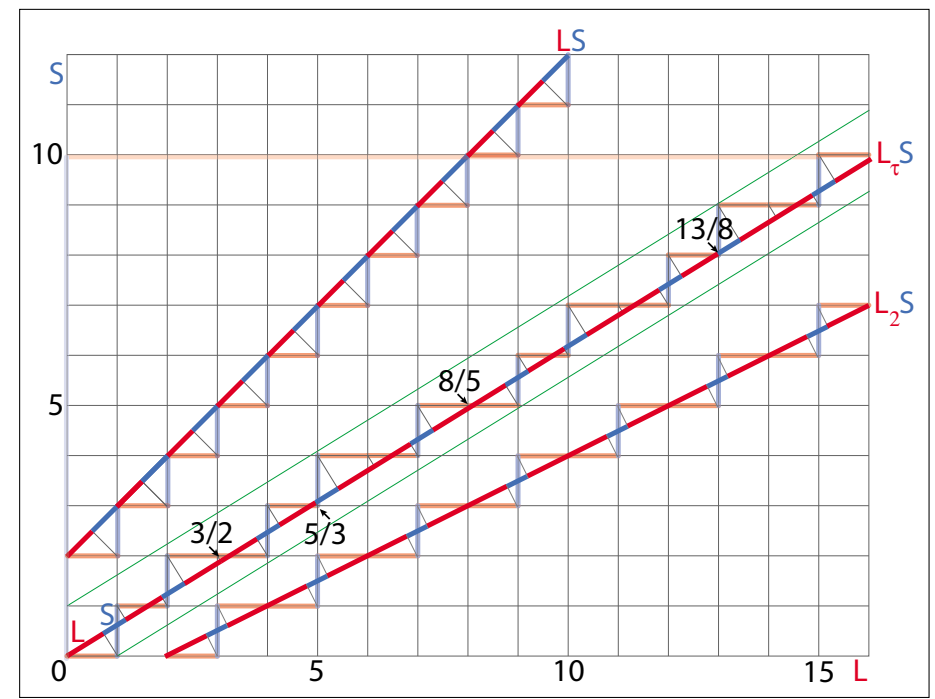

Fig. 4. The Fibonacci sequence (FS), consisting of short (S) and long (L) line segments, and two of its rational approximants in the 2D description. In the strip-projection method, all vertices of the $2 \mathrm{D}$ lattice inside the strip are projected onto a line (physical space) parallel to the strip boundary. Depending on the slope of the strip either the quasiperiodic FS or rational approximants are obtained. Note the different stoichiometries of the approximants $L S$ and $L_{2} S$ and the $F S L_{\tau} S$ where $\tau$ is the golden ratio $(1+\sqrt{5}) / 2=1.618$. 
periodic approximant structures LS and $\mathrm{L}_{2} \mathrm{~S}$ are generated or the quasiperiodic $\mathrm{FS}$ $\mathrm{L}_{\tau} \mathrm{S}$ depends only on the slope of the strip, which is defined by the overall stoichiometry $\mathrm{L}_{x} \mathrm{~S}(\tau$ is the golden ratio $(1+\sqrt{5}) / 2$ $=1.618)$. This implies that the algorithmic (Kolmogorow) complexity, i.e. the length of the algorithms for generating each of the three structures is exactly the same for the quasiperiodic as for the simplest periodic approximant structure (see ref. [26] and references therein). The situation changes if a structure with exactly the same number of sites per unit cell cannot be generated by the 2D-to-1D projection approach, or more generally an $n \mathrm{D}$ to $m \mathrm{D}$ projection $(n>m)$.

In contrast, the algorithmic entropy, which reflects the degree of randomness, can be larger in quasiperiodic than in periodic crystals. A structure, which is based on a tiling (quasilattice) of at least two different unit tiles, can show any kind of order of the unit tiles from full randomness to strict quasiperiodicity. In addition, the decoration (atomic arrangement) of the unit tiles may be disordered. In contrast periodic tilings (lattices) cannot show any disorder in the distribution of the unit cells, only in their decoration.

Now to the question, how do QCs grow, how does Mother Nature generate quasiperiodic order without knowing about the $n \mathrm{D}$-approach? The natural algorithm for QC-growth cannot be fundamentally different from that for periodic crystals; the same rules must apply as for complex intermetallics. Varying the chemical composition of an intermetallic phase in a particular binary or ternary system by a few percent can change its structure from periodic to quasiperiodic, from an approximant to a QC. Thus the driving force must be the chemical potential, provided that the constituting elements have the size ratios and valence electron concentrations needed for the constituting clusters. In terms of atomic interactions, potentials favoring two length scales in a proper ratio have been proven to be essential for the formation of the fundamental clusters and their assembly to quasiperiodic structures. Both periodic and quasiperiodic structures result as a compromise between the energetically best possible local atomic arrangement (AET, cluster) and the global densest packing of these units. Of course, at finite temperatures entropic contributions can also stabilize energetically less stable structures.

I want to conclude with a discussion of the scientific importance of the discovery of QCs. Is their crystallography so fundamentally different from that of periodic crystals that one can say their discovery induced a 'paradigm shift in crystallography' or are QCs just an exotic addendum to the ever increasing zoo of crystal structures? Or is quasiperiodic ordering simply a property of a state that is intermediate between the regular, periodic and the amorphous state as quite a few people think?

“... 'normal science' means research firmly based upon one or more past scientific achievements, achievements that some particular scientific community acknowledges for a time as supplying the foundation for its further practice.”[1, p. 10]

Kuhn introduced the term 'paradigm shift' in his book 'The structure of scientific revolution', published in 1962.[1] What is its signature? According to Kuhn it is a revolutionary, rather than an evolutionary, change in the conceptual basis and the fundamental tenets of a scientific field, i.e. a 'paradigm shift' is comparable to a phase transformation of first order rather than one of second order. As any other mature science, crystallography has a paradigm defining what 'normal science' in its field is and what contradicts it. In our case, 'normal crystallography' deals with periodic crystals, and the discovery of QCs induced an important change in the meaning of 'crystal'. Although the generic term remains 'crystal', one distinguishes now between 'periodic crystals' and 'aperiodic crystals'. The definition of 'crystal' is no longer based on the structure in direct space but on the properties of the Fourier spectrum (diffraction pattern) in reciprocal (Fourier) space. Accordingly, in 1992 the IUCr Interim Commission on Aperiodic Crystals suggested the following definition:[27] "In the following by 'crystal' we mean any solid having an essentially discrete diffraction diagram, and by 'aperiodic crystal' we mean any crystal in which three-dimensional lattice periodicity can be considered to be absent. As an extension, the latter term will also include those crystals in which three-dimensional periodicity is too weak to describe significant correlations in the atomic configuration, but which can be properly described by crystallographic methods developed for actual aperiodic crystals."

In other words, a periodic $n \mathrm{D}$ crystal structure has a Fourier spectrum with Fourier coefficients everywhere zero except at the points which are nodes of an $n \mathrm{D}$ reciprocal lattice. $m \mathrm{D}$ aperiodic crystal structures can be described as intersections of $n \mathrm{D}$ periodic crystal structures with the $m \mathrm{D}$ physical space $(n>m)$. In Fourier space, this corresponds to a projection of the $n \mathrm{D}$ reciprocal lattice onto the $m \mathrm{D}$ physical space leading to an $m \mathrm{D}$ Fourier module of rank $n$. If $m=3$ and $n>3$, then the crystal is aperiodic.

The change in the definition of a crystal is thus not revolutionary, it is simply an extension and generalization of the term. From the point of view of the underlying physics, there is no discontinuity between the description and understanding of QCs and 3D-periodic crystals; the same basic rules apply: only some parameters have to be adjusted. The biggest leap in our understanding of the solid state is the addition of the quasiperiodic equilibrium state to the long known periodic one. Whether the QC state is also a new ground state (stable at zero K) remains an open question.

In summary, the discovery of QC did not ring in a new crystallography; it extended the concept of higher-dimensional crystallography, which was already well established for incommensurately modulated phases and composite structures. From this point of view, it was not a paradigm shift for crystallography. It was a kind of small scientific revolution, however in the way crystallographically educated scientists think. In particular the commonplace that 3D periodicity is a necessary condition for sharp Bragg reflections had to be revised and extended to include higher dimensions.

Received: November 7, 2013

[1] T. Kuhn, 'The Structure of Scientific Revolution', University of Chicago Press, USA, 1962.

[2] D. Shechtman, I. Blech, D. Gratias, J.W. Cahn, Phys. Rev. Lett. 1984, 53, 1951.

[3] D. Levine, P. J. Steinhardt, Phys. Rev. Lett. 1984, 53, 2477.

[4] A. L. Mackay, Physica A (Utrecht) 1982, 114, 609.

[5] W. Steurer, S. Deloudi, Acta Cryst. A 2008, 64,

[6] W. Steurer, S. Deloudi, 'Crystallography of Quasicrystals - Concepts, Methods and Structures', Springer Series in Materials Science 126, Springer, Heidelberg, Dordrecht, London, New York, 2009.

[7] W. Steurer, Angew. Chem. Intern. Ed. 2011, 50, 10775.

[8] K. Barkan, H. Diamant, R. Lifshitz, Phys. Rev. B 2007, 83, 172201.

[9] S. Förster, K. Meinel, R. Hammer, M. Trautmann, W. Widdra, Nature 2013, 502, 215.

[10] A. P. Tsai, Acc. Chem. Res. 2003, 36, 31.

[11] W. Steurer, S. Deloudi, Struc. Chem. 2012, 23, 1115

[12] W. Steurer, Philos. Mag. 2006, 86, 1105.

[13] C. L. Henley, M. de Boissieu, W. Steurer, Philos. Mag. 2006, 86, 1131.

[14] W. Steurer, J. Non-Cryst. Sol. 2004, 334, 137.

[15] T. Dotera, Israel J. Chem. 2011, 51, 1197.

[16] T. Dotera, J. Poly. Sci. B 2012, 50, 155.

[17] S. Fischer, A. Exner, K. Zielske, J. Perlich, S. Deloudi, W. Steurer, P. Lindner, S. Förster, Proc. Nat. Acad. Sci. USA 2011, 108, 1810.

[18] A. Haji-Akbari, M. Engel, S.C. Glotzer, J. Chem. Phys. 2011, 135, 94101.

[19] W. Steurer, D. Sutter-Widmer, J. Phys. D 2007, 40, R229.

[20] C. Hermann, Acta Cryst. 1949, 2, 139.

[21] L. Palatinus, G. Chapuis, J. Appl. Cryst. 2007, 40, 786.

[22] A. Yamamoto, Sci. Technol. Adv. Mater. 2008, 9, 013001.

[23] W. Steurer, Chem. Soc. Rev. 2012, 41, 6719.

[24] W. Steurer, T. Haibach, Acta Cryst. A 1999, 55, 48.

[25] S. Deloudi, W. Steurer, Acta Cryst. A 2012, 68, 266.

[26] E. Estevez-Rams, R. González-Férez, Z. Kristallogr. 2009, 224, 179.

[27] IUCr Ad Interim Commision on Aperiodic Crystals, Acta Cryst. A 1992, 48, 928. 